\title{
Anatomical Variations of Recurrent Laryngeal Nerve: The Danger in Thyroid Surgery
}

\author{
Fatogoma Issa Koné*, Siaka Soumaoro, Naouma Cissé, N’faly Konaté, Kassim Diarra, \\ Djibril Samaké, Kadidiatou Singaré, Boubacary Guindo, Samba Karim Timbo, \\ Mohamed Amadou Keita
}

Department of ENT—Head and Neck Surgery, Gabriel Toure University Hospital, Bamako, Mali

Email: *konefatogomaissa@yahoo.fr

How to cite this paper: Koné, F.I., Soumaoro, S., Cissé, N., Konaté, N., Diarra, K., Samaké, D., Singaré, K., Guindo, B., Timbo, S.K. and Keita, M.A. (2019) Anatomical Variations of Recurrent Laryngeal Nerve: The Danger in Thyroid Surgery. Surgical Science, 10, 11-15.

https://doi.org/10.4236/ss.2019.101002

Received: April 26, 2018

Accepted: January 11, 2019

Published: January 14, 2019

Copyright () 2019 by author(s) and Scientific Research Publishing Inc. This work is licensed under the Creative Commons Attribution International License (CC BY 4.0).

http://creativecommons.org/licenses/by/4.0/

\begin{abstract}
Objectives: We report two types of anatomical variations of the recurrent laryngeal nerve in two patients. Through these two patients we wanted to highlight our surgical approach of the recurrent nerve in an unusual position and to describe the surgical implication of these almost rare variations. Case report: patient aged 28 and 58 admitted for goiter. They underwent a right lobisthmectomy. Both recurrences were approached retrograde. The anatomical variations of the nerve concerned the non-recurrent laryngeal nerve in the first patient. In the second patient there were three anatomical variations, namely an extra laryngeal bifurcation of the nerve, a pre-vascular position of the nerve and a branch connecting the vagus nerve and the recurrent nerve. No recurrence nerve injury was noted. Conclusion: The anatomical variations of the nerve are numerous. A careful dissection is a guarantee of a good prognosis.
\end{abstract}

\section{Keywords}

Anatomical Variation, Recurrent Laryngeal Nerve, Surgery, Mali

\section{Introduction}

The thyroid surgery requires anatomy awareness of the front and side of the neck, pharynx, laryngeal structures and any anatomical variation on the recurrent nerve [1]. The anatomy of the lower laryngeal nerve makes thyroid surgery difficult for the surgeon and the patient [2]. The identification and dissection is complicated and delicate for the subsequent plurality of anatomical variations. These variations are sources of vulnerability of the recurrent nerve [2]. The different anatomical variations reported by the literature relate to the nerve itself 
and its course with neighborhood organs [1] [2] [3]. These are the extra laryngeal divisions of the nerve, the link of the nerve to the inferior thyroid artery and the recurrent non-recurrent nerve. These variations can also be related to thyroid pathology.

We report two types of anatomical variations of the recurrent laryngeal nerve in two patients. Through these two patients we wanted to highlight our surgical approach of the recurrent nerve in an unusual position and to describe the surgical involvement of these almost rare variations.

\section{Case Report}

\section{Case 1:}

A 38 years old woman admitted for plunging goiter. Except for goiter, the patient had no other symptoms. We performed a right loboisthmectomy. The approach of the nerve was performed retrograde (superior approach) way due to the plunging character of goiter. It allowed the detection of a non-recurrent laryngeal nerve type 1 recurrent nerve (Figure 1). No recurrent nerve injury was noted and anatomopathological examination concluded with colloidal adenoma. The outcome was good.

Case 2:

A 58 years old woman admitted for large goiter. We performed a right lobisthmectomy. The nerve was approached retrograde way. We noted three variations (Figure 2, Figure 3):

- An extra laryngeal bifurcation.

- A pre vascular nerve.

- A branch that connects the vagus nerve to the recurrent nerve passing over the lower thyroid artery. No recurrent nerve injury was noted and anatomopathological examination concluded with colloidal adenoma. The outcome was good.

\section{Discussion}

A recurrent nerve injury comes from several factors. The implication of the

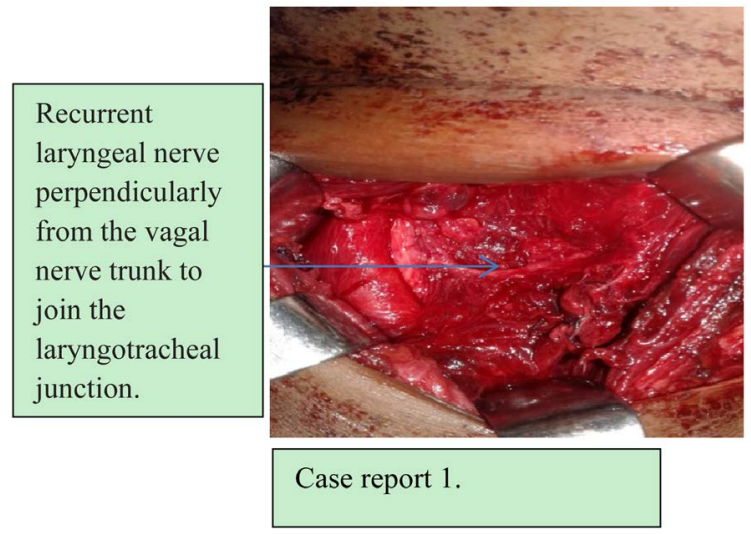

Figure 1. Non recurrent laryngeal nerve type 1. 


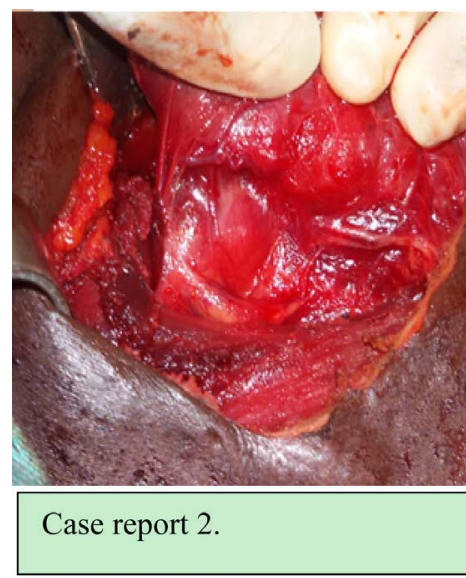

\section{Bifurcation of right recurrent laryngeal nerve}

Figure 2. Extralaryngeal bifurcation.

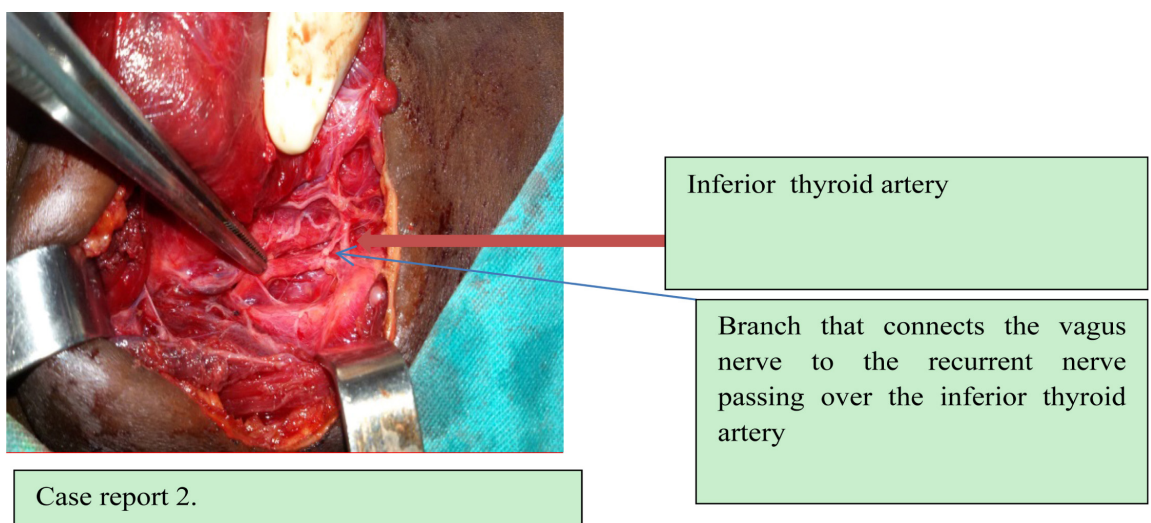

Figure 3. Anatomical variations between the laryngeal nerve and the lower thyroid artery.

surgeon's experience and the anatomical variations recognized by the recurrent nerve, constitute a heavy factor. These variations are most often fortuitous discovery intraoperatively as in our patients. The non-recurrent laryngeal nerve was revealed in our first patient intraoperatively. It is a rare entity with 0.25 0.99 percent [1]. In a series of Page C, 25 nerves on 251 nerves identified, no recurrent non-recurrent nerve was noted [4]. However it is a rare entity of discovery most often intraoperative. This anatomical variation exists most often on the right which explains it embryological development. This anatomical variation exists most often on the right which explains its embryological development. In rare cases of situ invertus he finds himself on the left. It derives from an embryology anomaly and is most often accompanied by the Lusoria artery responsible for dysphagia [1]. Apart from goiter no symptoms were identified in the patient. This condition is confined to dysphagia. Two mains paths are described for non-recurrent laryngeal nerve [1]:

- The type I arises perpendicularly from the vagal nerve trunk to join the laryngotracheal junction transversally via a short route.

- The type II is a low type of non-recurrent laryngeal nerve originates from the vagal nerve trunk and describes a supero-external concavity before reaching 
the tracheoesophageal groove. The nerve may meet the inferior thyroid artery.

Our first case we noted a non recurrent laryngeal type I in the right side. The anatomical relationship between the recurrent nerve and the inferior thyroid artery is highly variable at right and often left [4]. Similar to the recurrent non-recurrent nerve the variant identified in this study is on the right and is detached from the vagus nerve towards the non-recurrent nerve type II in the second patient. The three variations of the recurrent nerve reported in the second patient could be vulnerable at different levels. These variations of the recurrent laryngeal nerve are of significant surgical implication [5]. We have identified in the literature of multiple variations on the same nerve [2]. The surgical approach in case of anatomical variations of the recurrent nerve remains little discussed because they are discovered intraoperatively. These surgical approaches of the nerve depend on the size of the goiter, the character of goiter and thyroid intervention [3] [5]. Emphasis should be placed on these approaches to the extent that variations are suspected during radiological exploration.

The discovery of non-recurrent laryngeal nerve was fortuitous intraoperatively. It may be suspected [1]:

- Before the presence of Lusoria artery.

- When the nerve is not discovered in normal situation through the inferior thyroid artery.

Our superior approach through the unusual cricopharyngeal muscle was illustrated by the plunging character of goiter and the difficulty of externalizing the gland on the midline. The exteriorization of the gland could cause a stretch. Our first superior approach principle is corollary to the topographic situation of the gland and the constant concern to preserve the functionality of the recurrent nerve. It is admitted by certain author [3] [5]. The discovery of the non-recurrent nerve by this tract first was fortuitous. It may be convenient in situations where the non-recurrence is suspected by paraclinical elements.

The volume of the gland has been a factor encouraging us to approach the nerve by retrograde tract. No coagulation was performed at this level without visualization of the recurrent nerve. The discovery of a small branch was a factor encouraging us to look for a second branch as dictated by the literature [5]. The continuation of the dissection brought us to a thyroid artery in pre vascular position with a nerve branch. The surgical involvement of multiple anatomical variations is well known. The risk of nerve damage is high in the presence of extra laryngeal division [3] [4]. A dissection puncture remaining superficial to the nerve allowed us to discover these multiple variations without recurrent lesions.

\section{Conclusion}

The anatomical variations of the recurrent nerve are numerous and most often the discovery is done in intraoperative discovery. The operator should always bear in mind the presence of anatomical variation during thyroid surgery. Whi- 
chever approach is first; the surgeon's caution and experience avoids recurrent injury even in the presence of unusual anatomical variations of the recurrent nerve.

\section{Consent}

Written informed consent for publication.

\section{Funding}

No funding was utilized for this project.

\section{Conflicts of Interest}

The authors have no conflicts of interest.

\section{References}

[1] Page, C., Monet, P., Peltier, J., Bonnaire, B. and Strunski, V. (2008) Non-Récurrent Laryngeal Nerve Related to Thyroid Surgery: Report of Three Cases. The Journal of Laryngology \& Otology, 122, 757-761. https://doi.org/10.1017/S0022215107008389

[2] Shao, T.-L., Qiu, W.-H., Shen, B.-Y. and Yang, W.-P. (2015) New Variation of Right Recurrent Laryngeal Nerve: Cases Report. Indian Journal of Surgery, 77, 38-39.

[3] Page, C., Peltier, J., Charlet, L., Laude, M. and Strunski, V. (2006) Superior Approach to the Inferior Laryngeal Nerve in Thyroid Surgery: Anatomy, Surgical Technique and Indications. Surgical and Radiologic Anatomy, 28, 631-636. https://doi.org/10.1007/s00276-006-0141-9

[4] Page, C., Foulon, P. and Strunski, V. (2003) The Inferior Laryngeal Nerve: Surgical and Anatomic Considerations. Report of 251 Thyroidectomies. Surgical and Radiologic Anatomy, 25, 188-191. https://doi.org/10.1007/s00276-003-0129-7

[5] Guerrier, B., Zanaret, M.C., Guy, L.E. and Santini, J. (2006) Les differents types de chirurgie. In: Chirurgie de la thyroïde et de la parathyrö̈de, Amplifon, Italy, 57-132. 